\title{
Detection of cysteine-dependent Escherichia coli in a public health laboratory setting in Hong Kong
}

Cysteine-dependent Escherichia coli (CDEC) has been reported to cause septicaemia, probably through urinary tract infection, in Hong Kong and elsewhere (Tapsall \& McIver, 1986; Yuen et al., 1990). Its requirement for cysteine to grow has rendered it relatively difficult to be detected routinely in urine specimens. In an attempt to assess the contribution of CDEC to the apparently sterile pyuria cases in our clinical settings, a study was undertaken between April and December 2006 at the Microbiology Division, Public Health Laboratory Centre, Hong Kong. Our division is a public health diagnostic microbiology laboratory receiving clinical specimens mainly from outpatient clinics all over Hong Kong and from a regional public hospital. Urine samples with an appreciable degree of pyuria ( $>10$ white blood cells $\mu \mathrm{l}^{-1}$ ) were routinely cultured for pathogens using the commercial chromogenic agar medium CPS ID3 (bioMérieux) and Columbia blood agar. The culture plates were aerobically incubated at $35{ }^{\circ} \mathrm{C}$ and the samples were kept at $4{ }^{\circ} \mathrm{C}$. The next day, if no pathogen was isolated on the two culture plates, the corresponding sample would then be retrieved and plated onto cysteine-lactose-electrolyte-deficient (CLED) agar. This was done in case CPS ID3 was nutritionally insufficient for CDEC to grow. All the culture plates would then be incubated for a further $24 \mathrm{~h}$ before examination. Any E. coli isolates on any of the culture plates would be subjected to further characterization, including analysis of cysteine dependence. Within the 8 months of the study, approximately 33000 urine specimens were processed and around 5100 specimens were positive (15.4\%) for pathogens, of which $69 \%$ yielded E. coli. However, no E. coli was isolated on the CLED agar medium from a total of 500 cases of sterile pyuria following the above strategy despite the previous allegation that CLED agar may be a better culture medium for the isolation of CDEC (Sanyal, 1987). The absence of E. coli on the CLED agar could be due to the genuinely low incidence of CDEC among our patients. On the other hand, CPS ID3 agar may already be adequate for detection of CDEC. In fact, semi-quantitative growth experiments with serial 10-fold dilutions of a known cysteine- and thiamine-requiring E. coli strain, ATCC 23792, have shown CPS ID3 to be capable of recovering the strain with less than $1 \log$ difference as compared with nutrient agar.

In summary, our data supported the applicability of our strategy of culturing for urinary pathogens using the chromogenic agar medium CPS ID3, and CDEC is unlikely to be a significant reason for apparent sterile pyuria in our patients. This is in accord with a previous study in which CDEC appeared to be rare among acute urinary tract infections (Sanyal, 1987). Nevertheless, vigilance for its possible occurrence should be maintained with appropriate clinical management and microbiological testing strategy in hospitalized, elderly and debilitated patients, especially those with indwelling urinary catheters, as these are the groups of patients where recovery of CDEC has been reported most commonly.

\section{Yiu Wai Chu, Amy K. F. Chan, Grand K. L. Tsang and Janice Y. C. Lo}

Microbiology Division, Public Health Laboratory Services Branch, Centre for Health Protection, Department of Health, 382 Nam Cheong Street, Kowloon, Hong Kong SAR, PR China

Correspondence: Yiu Wai Chu (alf@chp.gov.hk)

\section{References}

Sanyal, D. (1987). Incidence of cysteine dependent Escherichia coli in a general practice population. J Clin Pathol 40, 930-933.

Tapsall, J. W. \& Mclver, C. J. (1986). Septicaemia caused by cysteine-requiring isolates of Escherichia coli. J Med Microbiol 22, 379-382.

Yuen, K. Y., Seto, W. H., Tsui, K. H. \& Hui, W. T. (1990). Septicemia caused by cysteinedependent Escherichia coli. J Clin Microbiol 28, 1047-1048. 